\title{
Heterologous Prime-Boost Enhances the Antitumor Immune Response Elicited by Plant-Virus-Based Cancer Vaccine
}

\author{
Hui Cai ${ }^{\dagger, \ddagger}$, Sourabh Shukla ${ }^{\dagger, \ddagger}$, Chao Wang ${ }^{\dagger, \ddagger}$, Hema Masarapu ${ }^{\S}$, Nicole F. Steinmetz ${ }^{\star}, \dagger, \ddagger, \|, \perp, \#$ \\ tDepartment of NanoEngineering, University of California, San Diego, La Jolla, California 92093, \\ United States \\ "Department of Radiology, University of California, San Diego, La Jolla, California 92093, United \\ States \\ ${ }^{\perp}$ Department of Bioengineering, University of California, San Diego, La Jolla, California 92093, \\ United States \\ \#Moores Cancer Center, University of California, San Diego, La Jolla, California 92093, United \\ States \\ FDepartment of Biomedical Engineering, Case Western Reserve University, 10900 Euclid Avenue, \\ Cleveland, Ohio 44106, United States \\ §Department of Virology, Sri Venkateswara University, Tirupati-517 502, Andhra Pradesh, India
}

\section{Abstract}

New cancer vaccine strategies are required to vanquish the self-tolerance and elicit robust immune responses against tumor-associated antigens and/or neoantigens. Contemporary approaches in nanomedicine center on the use of a single nanocarrier modified with multiple copies of multiple different functional domains, e.g., epitopes for vaccines. Therefore, we set out to develop a combinatorial approach toward the next-generation concept of epitope delivery: a prime-boost strategy in which the same epitope is delivered using different nanocarriers. We tested this concept in the setting of HER $2^{+}$breast cancer. We synthesized HER2-based cancer vaccines using three icosahedral plant viruses as carriers and evaluated the immune response as a result of repetitive, homologous immunization using BALB/c mice. Two of the vaccines induced a Th2-predominant response and the other a Th1-predominant response. To enhance the immunogenicity of the vaccines, we developed a heterologous prime-boost strategy with each of the vaccines administered only once, yielding higher titers of HER2-specific immunoglobulins and increasing the toxicity of the antisera toward cancer cells. The prime-boost also induced a Th1-predominant response. An in vivo tumor challenge showed that the prime-boost regimen reduced tumor growth and improved survival in mice. This novel strategy to elicit robust immune responses against

\footnotetext{
*Corresponding Author: nsteinmetz@ucsd.edu.

Supporting Information

The Supporting Information is available free of charge on the ACS Publications website at DOI: 10.1021/jacs.9b01523.

Figures S1-S6, Tables S1-S3, and experimental methods (PDF)

The authors declare no competing financial interest.
} 
weakly immunogenic antigens in principle could be broadly applicable to cancers and other diseases.

\section{Graphical Abstract}

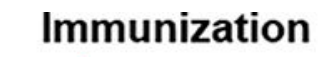

Immune response
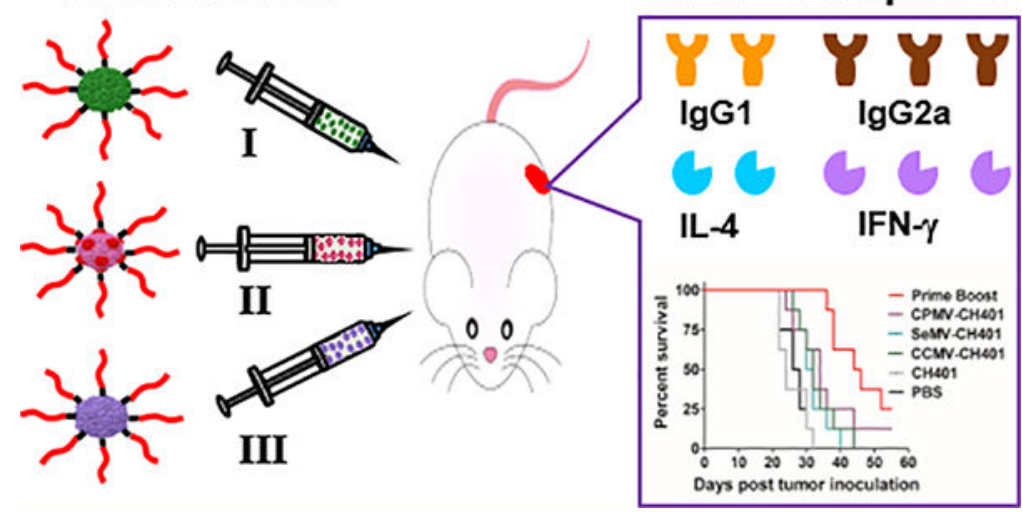

\section{INTRODUCTION}

Human epidermal growth factor receptor 2 (HER2) is a ligandless receptor tyrosine kinase that is typically amplified in breast, gastric, and esophageal cancer. ${ }^{1}$ The overexpression of HER2 mediates proliferative and anti-apoptotic signals, thus promoting an aggressive clinical course and a poor overall prognosis. ${ }^{2,3}$ Accordingly, HER2 immunotherapy has dramatically increased survival rates in patients with HER $2^{+}$breast cancer, ${ }^{4,5}$ particularly those treated with the monoclonal antibodies trastuzumab (Herceptin) and pertuzumab (Perjeta) $)^{6,7}$ combined with neoadjuvant chemotherapy. ${ }^{8-10}$ This suggests that HER2 overexpressed on cancer cells can be recognized and blocked by anti-HER 2 antibodies, and immunization strategies that produce HER2-targeting antibodies could also be effective treatment options. Indeed, an anti-HER2 DC1 vaccination in a phase I trial induced tumorspecific T-cell responses in patients with HER $2^{+}$breast cancer and also showed promise in early-stage breast cancer. ${ }^{11}$

HER2 is a self-antigen, so one challenge for immunization is to abolish self-tolerance and amplify the immune response. A promising strategy is the conjugation of HER2-derived Bcell epitope peptides to an antigenic carrier to break immune tolerance and induce antibodies that recognize HER2 on cancer cells. ${ }^{12}$ Virus nanoparticles (VNPs) based on plant viruses are safe and highly immunogenic vaccine delivery platforms that can induce potent and long-lasting immune responses in the absence of extra adjuvants by efficiently targeting antigen-presenting cells, priming innate and adaptive immune responses, and cross-linking specific receptors on B cells. ${ }^{13-16}$ Also, minimally immunogenic tumor-associated carbohydrate antigens have been conjugated to VNPs, thus enhancing the antitumor immune response. ${ }^{17,18}$ We have previously used the icosahedral cowpea mosaic virus (CPMV) and filamentous potato virus X (PVX) as carriers to deliver the CH401 peptide, ${ }^{19}$ a B-cell epitope from extracellular domain of human HER2. ${ }^{20}$ Following the repetitive immunization of mice, immunological evaluation demonstrated that the CPMV carrier elicited a higher 
titer of HER2-specific antibodies with the ability to recognize HER2 ${ }^{+}$cancer cells than the PVX carrier, suggesting that icosahedral plant virus particles are more efficient for epitope presentation. However, VNPs are usually immunogenic, and immune responses targeting the capsid proteins of the VNPs may be elicited, suppressing the immune response against the epitopes they carry. ${ }^{21}$

To focus the immune responses on the epitopes, we developed a heterologous prime-boost strategy in which different VNP carriers present the same epitope and each vaccine candidate is administered just once. During vaccination, the immune system is exposed to the same epitope but each time is activated by different carriers. This strategy is different from the heterologous prime-boost used to elicit broadly neutralizing antibodies against infectious diseases, ${ }^{22,23}$ where several strains of inactive virus (hence with different epitopes) are used to boost the immune response. ${ }^{24}$

Accordingly, we used three different VNPs based on plant viruses to deliver the rat CH401 epitope: CPMV, cowpea chlorotic mottle virus (CCMV), and Sesbania mosaic virus (SeMV). We compared the efficacies of repeated vaccination and the prime-boost strategy by immunizing $\mathrm{BALB} / \mathrm{c}$ mice and further by conducting an in vivo tumor challenge study in a DDHER2 murine model to develop an effective heterologous prime-boost strategy that enhanced the immune response against HER2. As the DDHER2 mouse cell line expresses the rat HER2 protein, the $\mathrm{CH} 401$ epitope derived from rat HER2 was selected in this study. Nevertheless, we confirmed that the antibodies elicited by this epitope also cross-bind to human HER2.

\section{RESULTS}

\section{Immunogenicity of CCMV, CPMV, and SeMV.}

We selected three plant viruses (CCMV, CPMV, and SeMV) to deliver the HER2 epitope. In each case, the VNPs assemble from the virus RNA and multivalent capsid proteins to form similar icosahedral capsids with diameters of $\sim 30 \mathrm{~nm}$ (Figure 1a). To determine their inherent immunogenicity, we added each of the VNPs to in vitro cultures of bone-marrowderived dendritic cells (BMDCs) harvested from BALB/c mice. After $18 \mathrm{~h}$, the secreted proinflammatory cytokines (IL- $1 \beta$, IL-6, IL-12, IFN- $a$, IFN- $\gamma$, and TNF- $a$ ) were measured by enzyme-linked immunorsorbent assay (ELISA) (Figure 1b). CPMV induced the secretion of all of the cytokines tested in our assay, but BMDCs treated with CCMV or SeMV secreted only a subset. Specifically, CCMV induced the secretion of IL-12, IFN- $a$, and IFN- $\gamma$, whereas SeMV induced the secretion of IL-12, IFN- $\gamma$, and IL-6. These data suggest that CPMV may be more broadly immunogenic than the other VNPs, but all three are immunogenic, and the cytokine profiles indicate that each is suitable for epitope presentation.

\section{Synthesis and Characterization of HER2-VNP Vaccines.}

The three VNPs form icosahedral capsids with similar symmetry ( $T 3$ for CCMV and SeMV, $p T 3$ for CPMV) and offer surface lysine side chains for chemical modification. Here we used copper-catalyzed azide-alkyne cycloaddition (CuAAC, click chemistry) to covalently 
conjugate the CH401 epitope (YQDMVLWKDVFRKNNQLAPV, derived from rat HER2 protein amino acid residues 167-186), which was prepared by solid-phase peptide synthesis (Figure 2a). A flexible GPSL linker sequence was placed at the C-terminus following a Lys $\left(\mathrm{N}_{3}\right)$ residue that provides the azide group for CuAAC. We also modified the VNPs with an NHS-alkyne linker and then conjugated them with $\mathrm{CH} 401$ in the presence of $\mathrm{Cu}(\mathrm{I})$. The reaction was complete within $45 \mathrm{~min}$. Significant aggregation and particle disassembly was observed when the reaction time was prolonged. The unconjugated peptides together with the additives for CuAAC were removed by centrifugal filtration, yielding the pure VNPHER2 vaccines CCMV/CPMV/SeMV-CH401 (Figure 2b).

We evaluated the conjugation efficiency by SDS-PAGE. A second, higher-molecular-weight band (CP-CH401) was clearly present in addition to each unmodified capsid protein (CP) band (Figure 2c). The intensities of these bands revealed the number of epitopes conjugated to each particle: approximately 41,36 , and 39 CH401 peptides were present on the CCMV, CPMV, and SeMV particles, respectively. Next, size-exclusion chromatography (SEC) was used to evaluate whether the VNPs remained intact after conjugation (Figure 2d). The VNPCH401 particles formed a single peak in the SEC profiles with an elution volume of $\sim 10$ $\mathrm{mL}$, similar to the unmodified wild-type VNPs (Table S1). The ratios of the absorbances at 260 and $280 \mathrm{~nm}$ (A260:A280) for the conjugated and wild-type VNPs were similar, indicating that the particles were intact and contained RNA (Table S1). The presence of virus RNA was confirmed by native agarose gel electrophoresis (Figure S1). The size of the VNP-CH401 particles was determined with greater precision by dynamic light scattering (DLS) (Figure 2e) and transmission electron microscopy (TEM) (Figure 2f). Both measurements confirmed that the size of the VNP-CH401 particles was similar to that of the wild-type particles, with a diameter of 28-30 nm. Therefore, CuAAC achieved successful conjugation of the HER2 epitope CH401 to CCMV, CPMV, and SeMV. The VNP-CH401 vaccine candidates were similar in structure and carried similar numbers of peptides on the particle surface, allowing the direct comparison of their immunization efficacies.

\section{Immunological Evaluation of VNP-CH401 Vaccines.}

Following the successful preparation of the VNP-CH401 vaccine candidates, we evaluated the immune response in mice. In many cases, multiple immunizations (prime-boost) are required for vaccines to be successful, and typically a homologous format is used (i.e., the priming and booster vaccines are the same). However, a heterologous format may be more effective, so we decided to test both strategies with our VNP-based vaccine candidates (Figure 3a). In the homologous (repeated vaccination) approach, female BALB/c mice ( $n=$ 11 per group) were subcutaneously immunized three times at intervals of 2 weeks with CCMV-CH401, CPMV-CH401, or SeMV-CH401 (100 $\mu \mathrm{g}$ per immunization), and a control group was immunized with the unconjugated $\mathrm{CH} 401$ peptide ( $2 \mu \mathrm{g}$ per immunization). For the heterologous (prime-boost) approach, mice were immunized with CCMV-CH401, CPMV-CH401, and SeMV-CH401 in sequence, which was randomly selected. One week after the third immunization, sera were collected and pooled together to evaluate the antibody responses. 
We first evaluated the total immunoglobulin $\mathrm{G}(\mathrm{IgG})$ against the $\mathrm{CH} 401$ peptide. The free peptide is nonimmunogenic, and accordingly, no $\mathrm{IgG}$ was raised against $\mathrm{CH} 401$ alone (Figure 3b). Conjugating the peptide to the VNPs enhanced its immunogenicity, as all three VNP-CH401 candidates induced significant anti-CH401 IgG responses after repeated vaccination. However, the three candidates elicited different responses in terms of antibody titers. ELISA profiles revealed that CCMV-CH401 achieved the strongest response, followed by SeMV-CH401 and CPMV-CH401 (Figure 3b). This was supported by the end-point titers (CPMV-CH401, 3200; SeMV-CH401, 6400; CCMV-CH401, 25 600) against the CH401 peptide (Table S2). Interestingly, the anti-CH401 IgG response induced by the prime-boost was significantly stronger than that induced by repeated vaccination. The end-point titer of the prime-boost was 51 200, which was double that of CCMV-CH401 alone, 8-fold higher than that of SeMV-CH401 alone, and 16-fold higher than that of CPMV-CH401 alone. Although the mice in each group were immunized with the same batch of VNP-CH401 vaccine, it is possible that individual mice showed distinct responses. To assess whether differences in antibody titers may correlate with survival, we also analyzed the IgG response for each individual mouse in each group at a serum dilution of 1:1600. Differences in antibody titers were noted, and the mouse with the longest survival time generally had a strong antibody response (Figure S3), although the highest antibody titer did not necessarily correlate with the longest mice survival (nevertheless, it should be noted that overall there was little variation among the individuals with high antibody titers).

VNPs are also immunogenic, so antibodies could be elicited to target the particle rather than the peptide, which would not be related to an antitumor immune response. We investigated whether the prime-boost strategy could mitigate the immune response against the VNPs, which was anticipated because each VNP was only injected once in the prime-boost regimen but three times in the repeated vaccination. VNP-coated plates were therefore tested by ELISA to detect VNP-specific antibodies. In the repeated vaccination, all three VNP-CH401 candidates induced a very strong IgG response against the virus $\mathrm{CP}$ (Figure $3 \mathrm{c}-\mathrm{e}$ ), with very high titers of 51200 (CCMV-CH401, SeMV-CH401) and 102400 (CPMV-CH401) against the corresponding VNPs (Table S2). As we expected, the prime-boost induced significantly weaker IgG responses against the VNPs. The anti-CCMV IgG titer generated by repeated vaccination with CCMV-CH401 was 8-fold higher than that for the prime-boost (6400), whereas a 2-fold increase in titer was observed for the other VNPs (Table S2). We concluded that the VNP-CH401 vaccines in the prime-boost regimen were more immunogenic in the context of the $\mathrm{CH} 401$ epitope than repeated vaccination, eliciting more $\mathrm{CH} 401$-specific IgG while mitigating the strong immune response toward the carriers by simply minimizing repeat exposure.

Next we evaluated the ability of sera from vaccinated animals to bind cancer cells expressing HER2, specifically the mouse breast cancer cell line DDHER2 and the human breast cancer cell line SKBR3, which express rat and human HER2 protein, respectively. We observed similar binding to both cell lines (Figure 3f,g). The sera induced by repeated vaccination with CCMV-CH401 bound slightly more strongly than sera induced by the other two vaccine candidates, but the prime-boost sera were the strongest of all. These results were consistent with the total IgG measured by ELISA (Figure 3b). Given the ability of the sera to bind cancer cells, we used an MTT assay to evaluate whether the sera induced complement- 
dependent cytotoxicity (CDC). In line with the IgG ELISA and cell-binding assays, all of the sera from vaccinated animals induced CDC, and the prime-boost sera exhibited much stronger cytotoxicity toward DDHER2 and SKBR3 cancer cells (Figure 3h,i). However, the sera induced by repeated immunization with CPMV-CH401 showed greater cytotoxicity toward both cell lines even though the IgG titer was lower than for the other VNP-CH401 candidates, suggesting that the three vaccines may elicit different antibody responses. Antibody-dependent cellular cytotoxicity (ADCC) is another important mode of cancer cell killing by antibodies. Although not investigated in this study, the antibodies induced by prime-boost, which exhibited enhanced binding to cancer cells expressing HER2, may also enhance the ADCC activity.

To gain insight into the specific antibody responses induced by each vaccine candidate, we determined the antibody isotypes (Figure 4) and their titers (Table S3) using the pooled serum. We also analyzed IgG1 and IgG2a responses for each individual mouse in each group at a serum dilution of 1:1600 (Figures S4 and S5). The free peptide CH401 induced only IgM, confirming its weak immunogenicity (Figure 4a). Repeated vaccination with CCMVCH401 induced predominantly IgG1 and IgG2a, with some IgG2b, IgG3, and IgM. However, IgG1 was significantly more abundant than IgG2a. The antibody isotypes induced by SeMV-CH401 were similar, but the titers were lower (Figure 4d). Interestingly, the antibody titers induced by CPMV-CH401 were also low, but the predominant antibody isotype was $\mathrm{IgG} 2 \mathrm{a}$ rather than $\mathrm{IgG1}$ (Figure 4c). The $\mathrm{IgG} 2 \mathrm{a} / \mathrm{IgG} 1$ ratio is related to the balance of Th1/Th2 cell responses, with ratios $>1$ indicating a Th1 response. ${ }^{25}$ As shown in Figure 4f, repeated vaccination with CPMV-CH401 induced a Th1 response whereas CCMV-CH401 and SeMV-CH401 induced a Th2 response, probably explaining the greater cytotoxicity of CPMV-CH401 despite the lower IgG titer compared with CCMV-CH401 and SeMV-CH401. The prime-boost strategy also induced abundant IgG1 and IgG2a, but again there was a high $\mathrm{IgG} 2 \mathrm{a} / \mathrm{IgG} 1$ ratio similar to that achieved by repeated vaccination with CPMV-CH401 (Figure 4e). The prime-boost strategy combined the high IgG titers and the high IgG2a/IgG1 ratio, with a 4-fold increase in IgG2a titers relative to repeated vaccination with CCMV-CH401 and an 8-fold increase relative to CPMV-CH401 and SeMV-CH401. We therefore concluded that the prime-boost regimen also induced a Th1 response, even though CPMV-CH401 was injected only once, suggesting that CPMV has a dominant effect on the Th1/Th2 balance.

To evaluate the immune responses induced by the VNP-CH401 vaccines in more detail, we collected splenocytes from three mice in each treatment group 7 days after the last immunization and analyzed their cytokine profiles. The three mice were randomly selected, and their antibody responses were analyzed at a serum dilution of 1:1600 (Figures S3-S5). First we tested for IL-4 (Th2) and IFN- $\gamma$ (Th1) by culturing splenocytes with or without the CH401 peptide. When stimulated with the peptide, splenocytes from mice repeatedly vaccinated with CCMV-CH401 or SeMV-CH401 secreted higher levels of IL-4 than unstimulated splenocytes, and the IL-4 level was significantly higher compared with the control group repeatedly vaccinated with the $\mathrm{CH} 401$ peptide, but no corresponding increase was seen in the CPMV-CH401 vaccination (Figure 5a). However, higher levels of IL-4 were also produced by stimulated splenocytes from the prime-boost treatment group, suggesting that all of the candidate vaccines except CPMV-CH401 induced a Th2 response. Stimulated 
splenocytes representing all groups secreted higher levels of IFN- $\gamma$, but the increase was more significant in mice repeatedly vaccinated with CPMV-CH401 or the prime-boost regimen, indicating a Th1 response (Figure 5b). Following stimulation with the $\mathrm{CH} 401$ peptide, we also analyzed the relative abundance of $\mathrm{CD} 4^{+} / \mathrm{IFN}-\gamma^{+}$and $\mathrm{CD} 8^{+} / \mathrm{IFN}-\gamma^{+} \mathrm{T}$ cells by flow cytometry (Figure S6). The abundance of $\mathrm{CD}^{+} / \mathrm{IFN}-\gamma^{+}$cells increased in the groups repeatedly vaccinated with CCMV-CH401 and prime-boost (Figure S6a). The abundance of $\mathrm{CD} 8^{+} / \mathrm{IFN}-\gamma^{+}$cells increased in the group repeatedly vaccinated with CPMVCH401, while a more significant increase was observed with the prime-boost strategy (Figure S6b). Together, these results confirm that CCMV-CH401 and SeMV-CH401 induced a Th2-predominant response targeting the CH401-HER2 epitope following repeated vaccination whereas CPMV-CH401 induced a primarily Th1 response. Interestingly, the prime-boost regimen elicited both Th1 and Th2 responses, but the Th1 response was predominant because more IgG2a was induced than IgG1 (Figure 4e) and the IFN- $\gamma$ level increased more than the IL-4 level (Figure 5). Our data also indicated that the Th1 response activated $\mathrm{CD} 8^{+} \mathrm{T}$ cells more efficiently (Figure S6b), suggesting that repeated vaccination with CPMV-CH401 and the prime-boost regimen are able to induce strong cytotoxic $\mathrm{T}$ lymphocyte (CTL) activities and establish a long-term-memory $\mathrm{CD} 8^{+} \mathrm{T}$ cell response and therefore may result in protective and therapeutic effects against HER2 ${ }^{+}$breast cancer.

\section{Antitumor Activity of VNP-CH401 Vaccine Candidates.}

To explore the therapeutic potential of the VNP-CH401 vaccine candidates, we next investigated their activity against HER2 ${ }^{+}$tumors in vivo in a DDHER2 murine model. ${ }^{26}$ Two weeks after the third immunization (Figure 6a), mice were subcutaneously inoculated with DDHER 2 cells in the right flank $\left(1 \times 10^{6}\right.$ cells/mouse, $\left.n=8\right)$, and the tumor volume was monitored every other day. Repeated vaccination with the CH401 peptide had no effect compared to nonimmunized controls. Repeated vaccination with each of the VNP-CH401 candidates led to similar but modest delays in tumor growth (Figure 6b). The average tumor volumes after 24 days were $1000 \mathrm{~mm}^{3}$ for the control and CH401 groups and $600 \mathrm{~mm}^{3}$ for the repeated-vaccination groups. This confirmed that the IgM response elicited by the peptide alone did not confer protection and that an $\operatorname{IgG}$ response is necessary for the vaccine to work. In support of the CDC data provided above (Figure 3g,h), repeated vaccination with CPMV-CH401 achieved antitumor activity comparable to those for the other two VNPs despite the lower titer of anti-CH401 IgG, suggesting that tumor growth was suppressed more effectively by the Th1 response. The prime-boost strategy resulted in a much stronger suppression of tumor growth than repeated vaccination (Figure 6b). The average tumor volume was $\sim 250 \mathrm{~mm}^{3} 24$ days post-challenge. The tumor growth data were supported by survival analysis, which showed that repeated vaccination only prolonged survival from 24 to 31-34 days, whereas the median survival was 45 days in the prime-boost group (Figure 6c). When all of the control animals had succumbed to the disease (day 32), $100 \%$ of the prime-boost group remained alive, compared with 38\%, 38\%, and 25\% in the groups repeatedly vaccinated with CCMV-CH401, CPMV-CH401, and SeMV-CH401, respectively. Prolonged survival was correlated with strong anti-CH401 antibody responses (Figures S3S5). These results showed that repeated vaccination with VNP-CH401 candidates induced modest in vivo protection against HER $2^{+}$cancer but that the heterologous prime-boost strategy was much more efficacious. Taken together with the antibody, cytokine, and $\mathrm{T}$ cell 
analyses, these results allow us to conclude that the Th1 immune response, which can induce predominant IgG2a, the secretion of IFN- $\gamma$, and the activation of $\mathrm{CD} 4^{+} / \mathrm{CD}^{+} \mathrm{T}$ cells, is more desired for efficient antitumor immunity.

\section{DISCUSSION}

VNPs based on plant viruses such as CCMV, CPMV, and SeMV are ideal carriers for the delivery of antigens because they are small enough to enter lymphatic vessels and are readily taken up by antigen-presenting cells. ${ }^{27,28}$ The viral RNA is a natural ligand for TLR7, which stimulates the immune system, ${ }^{29}$ and the repetitive multivalent protein structures are also immunogenic in the absence of additional TLR-mediated stimuli. ${ }^{30,31}$ Such VNPs can be used to deliver minimally immunogenic peptides, including self-antigens, ${ }^{17,18}$ raising the possibility that VNPs conjugated to the HER2-derived peptide epitope $\mathrm{CH} 401$ could be used to induce protective immune responses against HER2 ${ }^{+}$breast cancer. ${ }^{19,20}$ The principle of protective vaccination has been demonstrated, for example, by the observation that an antiHER2 dendritic cell vaccine induced a systemic immune response causing the regression of HER $2^{+}$ductal carcinoma. ${ }^{11}$ Nevertheless, engineering dendritic cells for vaccine applications is technologically challenging and costly, ${ }^{32}$ and therefore, there is a need for alternative technologies such as VNP prime-boost strategies.

It is important to characterize the immunogenicity of the carrier itself in order to develop a suitable immunization strategy that does not suppress the immune response against the conjugated antigen. ${ }^{21}$ Accordingly, we carried out a preliminary analysis of the immunogenicity of each virus in primary BMDC cultures, which revealed that all three particles stimulated the secretion of cytokines that regulate T-cell-mediated immune responses and the Th1/Th2 balance. ${ }^{33-35}$ We found that CPMV stimulated a broader response than CCMV and SeMV, consistent with our earlier studies, $, 36,37$ but these results showed that in principle CCMV, CPMV, and SeMV are suitable carriers for CH401. All three viruses are structurally well-characterized, and their potential for functionalization is understood. ${ }^{38-40}$ This allowed us to use lysine side chains on all three viruses for CuAAC chemical modification ${ }^{41,42}$ in the knowledge that the resulting 1,2,3-triazole structure is not inherently immunogenic. ${ }^{43,44}$ Structural analysis of the synthetic VNP-CH401 vaccine candidates revealed that the sizes and compositions of the particles were similar to those of the wild-type viruses, as expected.

To focus the immune responses on HER2, we developed a heterologous prime-boost strategy in which the three carriers, each presenting the same epitope, were delivered sequentially. To the best of our knowledge, this is the first example of a prime-boost strategy applied to deliver a single peptide epitope (as opposed to homologous prime-boost or heterologous prime-boost using distinct epitope ${ }^{24}$ ). We compared our new strategy to the traditional homologous serial vaccination approach in which the same VNP-CH401 candidate was administered three times. When the homologous strategy was used, all three VNP-CH401 candidates induced HER2-specific IgG, but the titers induced by CCMV-CH401 were 4- and 8-fold higher than those induced by SeMV-CH401 and CPMV-CH401, respectively. The heterologous prime-boost strategy elicited even higher IgG titers, double that of CCMVCH401. The serial homologous vaccination also induced high IgG titers against the carrier, 
but the effect was much weaker using the heterologous prime-boost strategy, confirming that the prime-boost approach focuses the immune response on the peptide.

Interestingly, the balance between Th1 and Th2 responses also differed among the different vaccine candidates. CCMV-CH401 and SeMV-CH401 induced a Th2-predominant response, whereas CPMV-CH401 induced a Th1-predominant response, as did the prime-boost strategy. This was addressed in more detail by analyzing the antibody isotypes, their cytotoxicities toward HER2 ${ }^{+}$cell lines, and the cytokine profiles of splenocytes isolated from immunized mice and stimulated with $\mathrm{CH} 401$. The results of all these experiments confirmed a model in which CCMV-CH401 and SeMV-CH401 induced a Th2-predominant response (more IgG1 than IgG2a, high levels of secreted IL-4 but low levels of IFN- $\gamma$ ) whereas CPMV-CH401 induced a Th1-predominant response (more IgG2a than IgG1, high levels of secreted IFN- $\gamma$ but low levels of IL-4). The heterologous prime-boost also induced a Th1-predominant response, but the levels of both IgG1 and IgG2a were higher than achieved using the homologous strategy, suggesting that both the Th1 and Th2 responses were stronger. Accordingly, we analyzed the $\mathrm{CD} 4^{+} / \mathrm{IFN}-\gamma^{+}$and $\mathrm{CD} 8^{+} / \mathrm{IFN}-\gamma^{+} \mathrm{T}$ cell profiles and found that $\mathrm{CD} 8^{+} / \mathrm{IFN}-\gamma^{+}$cells became more abundant following the homologous administration of CPMV-CH401 or the prime-boost regimen. Our results indicate that the predominant Th1 response is more efficient in activating the $\mathrm{CD} 8^{+} \mathrm{T}$ cells and also support the outcome of an earlier study in which mice were immunized with a MUC1 glycopeptide antitumor vaccine, where the resulting IgG2a-rich sera indicated a Th1-predominant response with enhanced cytotoxicity against cancer cells. ${ }^{43}$ The immunization order in our prime-boost regime was randomly assigned as CCMV-CPMV-SeMV. Thus, the immune system was primed by the CCMV-CH401 and then boosted by CPMV-CH401 and SeMVCH401. During vaccination, the initial priming events elicited by a first exposure to an antigen are usually imprinted on the immune system. ${ }^{45}$ Therefore, different orders in the prime-boost regime could result in distinct immunological outcomes, as the immunogenicities of the three VNPs are different. In particular, changing the order may influence the Th1/Th2 balance. It is interesting to note that the prime-boost immunization was characterized by a Th1/Th2 bias (see Figure 4), characteristic of the CPMV-CH401 formulation, which may be dominant and overwrite the Th2 bias of the CCMV-CH401 (given first) and SeMV-CH401 (administered last). It will be important to dissect these questions in future investigations.

The enhanced performance of the heterologous prime-boost strategy was also reflected by the improved in vivo antitumor activities in the DDHER2 breast cancer model. ${ }^{26}$ The mice in the heterologous prime-boost group survived longer than the other cohorts, and the tumors grew more slowly, although all of the mice eventually succumbed to the disease because it is a highly aggressive cancer model. These results showed that although repeated vaccination with the VNP-CH401 candidates induced modest protection against HER2 ${ }^{+}$tumors in vivo, the heterologous prime-boost strategy was much more efficacious because it elicited a strong and Th1-predominant immune response. Indeed, although the antibody response elicited by repeated CPMV-CH401 vaccination was weaker, stronger CDC cytotoxicity and longer animal survival were observed compared with those by CCMV-CH401 and SeMV-CH401, implying that the Th1 response is more efficacious for antitumor immunity. The secretion of Th1 cytokines such as IFN- $\gamma$ can promote the activity of $\mathrm{CD} 8^{+} \mathrm{T}$ cells and natural killer 
cells, thus helping to prevent tumor growth. ${ }^{46}$ An existing anti-HER2 Th1 response in healthy donors could thus confer protection, whereas the suppression of this immune response by the tumor could allow it to evade immune surveillance. ${ }^{47}$ In a previous study, the anti-HER 2 Th1 response was lost during breast tumorigenesis and was not readily reversed by standard therapies, correlating with the lack of a complete response to neoadjuvant therapy and reduced survival. ${ }^{48}$ Furthermore, the protective antitumor activity may also be partially contributed by ADCC, CTL, and phagocytosis; however, this has not been elucidated here. Nevertheless, our heterologous prime-boost strategy provides a new approach to induce systemic immunity against HER2 that could invigorate current research into the development of breast cancer vaccines. ${ }^{49-52}$

\section{Supplementary Material}

Refer to Web version on PubMed Central for supplementary material.

\section{ACKNOWLEDGMENTS}

This research was supported by a grant from the National Institutes of Health (R01 CA224605 to N.F.S.). H.M. acknowledges the United States-India Education Foundation (USIEF) for providing a Fulbright-Nehru Academic and Professional Excellence Fellowship (FBNAPE). The authors acknowledge Prof. Darrell Irvine (Koch Institute for Integrative Cancer Research at MIT, Cambridge, MA) for providing DDHER2 cancer cells.

\section{REFERENCES}

(1). Arteaga CL; Engelman JA ERBB receptors: from oncogene discovery to basic science to mechanism-based cancer therapeutics. Cancer Cell 2014, 25, 282-303. [PubMed: 24651011]

(2). Wang GS; Zhu H; Bi SJ Pathological features and prognosis of different molecular subtypes of breast cancer. Mol. Med. Rep 2012, 6, 779-782. [PubMed: 22797840]

(3). Bianchini G; Gianni L The immune system and response to HER2-targeted treatment in breast cancer. Lancet Oncol. 2014, 15, E58-E68. [PubMed: 24480556]

(4). Piccart-Gebhart MJ; Procter M; Leyland-Jones B; Goldhirsch A; Untch M; Smith I; Gianni L; Baselga J; Bell R; Jackisch C; Cameron D; Dowsett M; Barrios CH; Steger G; Huang CS; Andersson M; Inbar M; Lichinitser M; Lang I; Nitz U; Iwata H; Thomssen C; Lohrisch C; Suter TM; Ruschoff J; Suto T; Greatorex V; Ward C; Straehle C; McFadden E; Dolci MS; Gelber RD Herceptin Adjuvant Trial Study, T. Trastuzumab after adjuvant chemotherapy in HER2-positive breast cancer. N. Engl. J. Med 2005, 353, 1659-1672. [PubMed: 16236737]

(5). de Azambuja E; Holmes AP; Piccart-Gebhart M; Holmes E; Di Cosimo S; Swaby RF; Untch M; Jackisch C; Lang I; Smith I; Boyle F; Xu B; Barrios CH; Perez EA; Azim HA Jr.; Kim SB; Kuemmel S; Huang CS; Vuylsteke P; Hsieh RK; Gorbunova V; Eniu A; Dreosti L; Tavartkiladze N; Gelber RD; Eidtmann H; Baselga J Lapatinib with trastuzumab for HER2-positive early breast cancer (NeoALTTO): survival outcomes of a randomised, open-label, multicentre, phase 3 trial and their association with pathological complete response. Lancet Oncol. 2014, 15, 1137-1146. [PubMed: 25130998]

(6). Hudis CA Trastuzumab-mechanism of action and use in clinical practice. N. Engl. J. Med 2007, 357, 39-51. [PubMed: 17611206]

(7). Sabatier R; Goncalves A [Pertuzumab (Perjeta $\left.{ }^{\circledR}\right)$ approval in HER2-positive metastatic breast cancers]. Bull. Cancer 2014, 101, 765-771. [PubMed: 25091659]

(8). Gianni L; Eiermann W; Semiglazov V; Manikhas A; Lluch A; Tjulandin S; Zambetti M; Vazquez F; Byakhow M; Lichinitser M; Climent MA; Ciruelos E; Ojeda B; Mansutti M; Bozhok A; Baronio R; Feyereislova A; Barton C; Valagussa P; Baselga J Neoadjuvant chemotherapy with trastuzumab followed by adjuvant trastuzumab versus neoadjuvant chemotherapy alone, in patients with HER2-positive locally advanced breast cancer (the NOAH trial): a randomised 
controlled superiority trial with a parallel HER2-negative cohort. Lancet 2010, 375, 377-384. [PubMed: 20113825]

(9). Untch M; Rezai M; Loibl S; Fasching PA; Huober J; Tesch H; Bauerfeind I; Hilfrich J; Eidtmann H; Gerber B; Hanusch C; Kuhn T; du Bois A; Blohmer JU; Thomssen C; Dan Costa S; Jackisch C; Kaufmann M; Mehta K; von Minckwitz G Neoadjuvant treatment with trastuzumab in HER2positive breast cancer: results from the Gepar Quattro study. J. Clin. Oncol 2010, 28, 2024-2031. [PubMed: 20308670]

(10). Untch M; Fasching PA; Konecny GE; Hasmuller S; Lebeau A; Kreienberg R; Camara O; Muller V; du Bois A; Kuhn T; Stickeler E; Harbeck N; Hoss C; Kahlert S; Beck T; Fett W; Mehta KM; von Minckwitz G; Loibl S Pathologic complete response after neoadjuvant chemotherapy plus trastuzumab predicts favorable survival in human epidermal growth factor receptor 2overexpressing breast cancer: results from the TECHNO trial of the AGO and GBG study groups. J. Clin. Oncol 2011, 29, 3351-3357. [PubMed: 21788566]

(11). Lowenfeld L; Mick R; Datta J; Xu S; Fitzpatrick E; Fisher CS; Fox KR; DeMichele A; Zhang PJ; Weinstein SP; Roses RE; Czerniecki BJ Dendritic Cell Vaccination Enhances Immune Responses and Induces Regression of HER2(pos) DCIS Independent of Route: Results of Randomized Selection Design Trial. Clin. Cancer Res 2017, 23, 2961-2971. [PubMed: 27965306]

(12). Singer J; Manzano-Szalai K; Fazekas J; Thell K; Bentley-Lukschal A; Stremnitzer C; RothWalter F; Weghofer M; Ritter M; Pino Tossi K; Horer M; Michaelis U; Jensen-Jarolim E Proof of concept study with an HER-2 mimotope anticancer vaccine deduced from a novel AAVmimotope library platform. Oncoimmunology. 2016, 5, No. e1171446. [PubMed: 27622022]

(13). Roldao A; Mellado MC; Castilho LR; Carrondo MJ; Alves PM Virus-like particles in vaccine development. Expert Rev. Vaccines 2010, 9, 1149-1176. [PubMed: 20923267]

(14). Jegerlehner A; Storni T; Lipowsky G; Schmid M; Pumpens P; Bachmann MF Regulation of IgG antibody responses by epitope density and CD21-mediated costimulation. Eur. J. Immunol 2002, 32, 3305-3314. [PubMed: 12555676]

(15). Wen AM; Steinmetz NF Design of virus-based nanomaterials for medicine, biotechnology, and energy. Chem. Soc. Rev 2016, 45, 4074-4126. [PubMed: 27152673]

(16). Frietze KM; Peabody DS; Chackerian B Engineering virus-like particles as vaccine platforms. Curr. Opin. Virol 2016, 18, 44-49. [PubMed: 27039982]

(17). Miermont A; Barnhill H; Strable E; Lu X; Wall KA; Wang Q; Finn MG; Huang X Cowpea mosaic virus capsid: a promising carrier for the development of carbohydrate based antitumor vaccines. Chem. - Eur. J 2008, 14, 4939-4947. [PubMed: 18431733]

(18). Yin Z; Comellas-Aragones M; Chowdhury S; Bentley P; Kaczanowska K; Benmohamed L; Gildersleeve JC; Finn MG; Huang X Boosting immunity to small tumor-associated carbohydrates with bacteriophage qbeta capsids. ACS Chem. Biol 2013, 8, 1253-1262. [PubMed: 23505965]

(19). Shukla S; Myers JT; Woods SE; Gong X; Czapar AE; Commandeur U; Huang AY; Levine AD; Steinmetz NF Plant viral nanoparticles-based HER2 vaccine: Immune response influenced by differential transport, localization and cellular interactions of particulate carriers. Biomaterials 2017, 121, 15-27. [PubMed: 28063980]

(20). Miyako H; Kametani Y; Katano I; Ito R; Tsuda B; Furukawa A; Saito Y; Ishikawa D; Ogino K; Sasaki S; Imai K; Habu S; Makuuchi H; Tokuda Y Antitumor effect of new HER2 peptide vaccination based on B cell epitope. Anticancer Res. 2011, 31, 3361-3368. [PubMed: 21965747]

(21). Jegerlehner A; Wiesel M; Dietmeier K; Zabel F; Gatto D; Saudan P; Bachmann MF Carrier induced epitopic suppression of antibody responses induced by virus-like particles is a dynamic phenomenon caused by carrier-specific antibodies. Vaccine 2010, 28, 5503-5512. [PubMed: 20307591]

(22). Garrone P; Fluckiger AC; Mangeot PE; Gauthier E; Dupeyrot-Lacas P; Mancip J; Cangialosi A; Du Chene I; LeGrand R; Mangeot I; Lavillette D; Bellier B; Cosset FL; Tangy F; Klatzmann D; Dalba C A prime-boost strategy using virus-like particles pseudotyped for HCV proteins triggers broadly neutralizing antibodies in macaques. Sci. Transl. Med 2011, 3, 94ra71.

(23). Brown SA; Surman SL; Sealy R; Jones BG; Slobod KS; Branum K; Lockey TD; Howlett N; Freiden P; Flynn P; Hurwitz JL Heterologous Prime-Boost HIV-1 Vaccination Regimens in PreClinical and Clinical Trials. Viruses 2010, 2, 435-467. [PubMed: 20407589] 
(24). Lu S Heterologous prime-boost vaccination. Curr. Opin. Immunol 2009, 21, 346-351. [PubMed: 19500964]

(25). Stevens TL; Bossie A; Sanders VM; Fernandez-Botran R; Coffman RL; Mosmann TR; Vitetta ES Regulation of antibody isotype secretion by subsets of antigen-specific helper T cells. Nature 1988, 334, 255-258. [PubMed: 2456466]

(26). Moynihan KD; Opel CF; Szeto GL; Tzeng A; Zhu EF; Engreitz JM; Williams RT; Rakhra K; Zhang MH; Rothschilds AM; Kumari S; Kelly RL; Kwan BH; Abraham W; Hu K; Mehta NK; Kauke MJ; Suh H; Cochran JR; Lauffenburger DA; Wittrup KD; Irvine DJ Eradication of large established tumors in mice by combination immunotherapy that engages innate and adaptive immune responses. Nat. Med 2016, 22, 1402-1410. [PubMed: 27775706]

(27). Manolova V; Flace A; Bauer M; Schwarz K; Saudan P; Bachmann MF Nanoparticles target distinct dendritic cell populations according to their size. Eur. J. Immunol 2008, 38, 1404-1413. [PubMed: 18389478]

(28). Bachmann MF; Jennings GT Vaccine delivery: a matter of size, geometry, kinetics and molecular patterns. Nat. Rev. Immunol 2010, 10, 787-796. [PubMed: 20948547]

(29). Bessa J; Kopf M; Bachmann MF Cutting edge: IL-21 and TLR signaling regulate germinal center responses in a B cell-intrinsic manner. J. Immunol 2010, 184, 4615-4619. [PubMed: 20368279]

(30). Lizotte PH; Wen AM; Sheen MR; Fields J; Rojanasopondist P; Steinmetz NF; Fiering S In situ vaccination with cowpea mosaic virus nanoparticles suppresses metastatic cancer. Nat. Nanotechnol 2016, 11, 295-303. [PubMed: 26689376]

(31). Chackerian B; Lenz P; Lowy DR; Schiller JT Determinants of autoantibody induction by conjugated papillomavirus virus-like particles. J. Immunol 2002, 169, 6120-6126. [PubMed: 12444114]

(32). Van de Velde AL; Anguille S; Beutels P; Dom S; Cornille I; Nijs G; Van Tendeloo VF; Smits EL; Verlinden A; Gadisseur AP; Schroyens WA; Berneman ZN Cost Analysis of Immunotherapy Using Dendritic Cells for Acute Myeloid Leukemia Patients. Blood 2014, 124 (21), 1322.

(33). Macatonia SE; Hosken NA; Litton M; Vieira P; Hsieh CS; Culpepper JA; Wysocka M; Trinchieri G; Murphy KM; O'Garra A Dendritic cells produce IL-12 and direct the development of Th1 cells from naive CD4+ T cells. J. Immunol 1995, 154, 5071-5079. [PubMed: 7730613]

(34). Ramos HJ; Davis AM; George TC; Farrar JD IFN-alpha is not sufficient to drive Th1 development due to lack of stable T-bet expression. J. Immunol 2007, 179, 3792-3803. [PubMed: 17785816]

(35). Trevejo JM; Marino MW; Philpott N; Josien R; Richards EC; Elkon KB; Falck-Pedersen E TNFalpha -dependent maturation of local dendritic cells is critical for activating the adaptive immune response to virus infection. Proc. Natl. Acad. Sci. U. S. A 2001, 98, 12162-12167. [PubMed: 11593031]

(36). Lee KL; Murray AA; Le DHT; Sheen MR; Shukla S; Commandeur U; Fiering S; Steinmetz NF Combination of Plant Virus Nanoparticle-Based in Situ Vaccination with Chemotherapy Potentiates Antitumor Response. Nano Lett. 2017, 17, 4019-4028. [PubMed: 28650644]

(37). Murray AA; Wang C; Fiering S; Steinmetz NF In Situ Vaccination with Cowpea vs Tobacco Mosaic Virus against Melanoma. Mol. Pharm 2018, 15, 3700-3716. [PubMed: 29798673]

(38). Gillitzer E; Willits D; Young M; Douglas T Chemical modification of a viral cage for multivalent presentation. Chem. Commun 2002, 2390-2391.

(39). Canizares MC; Lomonossoff GP; Nicholson L Development of cowpea mosaic virus-based vectors for the production of vaccines in plants. Expert Rev. Vaccines 2005, 4, 687-697. [PubMed: 16221070]

(40). Bhuvaneshwari M; Subramanya HS; Gopinath K; Savithri HS; Nayudu MV; Murthy MR Structure of sesbania mosaic virus at 3 A resolution. Structure 1995, 3, 1021-1030. [PubMed: 8589997]

(41). Wang Q; Chan TR; Hilgraf R; Fokin VV; Sharpless KB; Finn MG Bioconjugation by copper(I)catalyzed azide-alkyne [3 + 2] cycloaddition. J. Am. Chem. Soc 2003, 125, 3192-3193. [PubMed: 12630856]

(42). Hong V; Presolski SI; Ma C; Finn MG Analysis and optimization of copper-catalyzed azidealkyne cycloaddition for bioconjugation. Angew. Chem., Int. Ed 2009, 48, 9879-9883. 
(43). Cai H; Sun ZY; Chen MS; Zhao YF; Kunz H; Li YM Synthetic multivalent glycopeptidelipopeptide antitumor vaccines: impact of the cluster effect on the killing of tumor cells. Angew. Chem. Int. Ed 2014, 53, 1699-1703.

(44). Cai H; Zhang R; Orwenyo J; Giddens J; Yang Q; LaBranche CC; Montefiori DC; Wang LX Multivalent Antigen Presentation Enhances the Immunogenicity of a Synthetic Three-Component HIV-1 V3 Glycopeptide Vaccine. ACS Cent. Sci 2018, 4, 582-589. [PubMed: 29806004]

(45). Woodland DL Jump-starting the immune system: prime-boosting comes of age. Trends Immunol. 2004, 25, 98-104. [PubMed: 15102369]

(46). Kim HJ; Cantor H CD4 T-cell subsets and tumor immunity: the helpful and the not-so-helpful. Cancer Immunol. Res 2014, 2, 91-98. [PubMed: 24778273]

(47). Datta J; Rosemblit C; Berk E; Showalter L; Namjoshi P; Mick R; Lee KP; Brod AM; Yang RL; Kelz RR; Fitzpatrick E; Hoyt C; Feldman MD; Zhang PJ; Xu S; Koski GK; Czerniecki BJ Progressive loss of anti-HER2 CD4(+) T-helper type 1 response in breast tumorigenesis and the potential for immune restoration. Oncoimmunology. 2015, 4, No. e1022301. [PubMed: 26451293]

(48). Nocera NF; Lee MC; De La Cruz LM; Rosemblit C; Czerniecki BJ Restoring Lost Anti-HER-2 Th1 Immunity in Breast Cancer: A Crucial Role for Th1 Cytokines in Therapy and Prevention. Front. Pharmacol 2016, 7, 356. [PubMed: 27766079]

(49). Ishihara Y; Harada M; Azuma K; Tamura M; Shomura H; Fujii T; Itoh K; Shichijo S HER2/neuderived peptides recognized by both cellular and humoral immune systems in HLA-A2+ cancer patients. Int. J. Oncol 2004, 24, 967-975. [PubMed: 15010837]

(50). Ladjemi MZ; Jacot W; Chardes T; Pelegrin A; Navarro-Teulon I Anti-HER2 vaccines: new prospects for breast cancer therapy. Cancer Immunol. Immunother 2010, 59, 1295-1312. [PubMed: 20532501]

(51). Clifton GT; Mittendorf EA; Peoples GE Adjuvant HER2/neu peptide cancer vaccines in breast cancer. Immunotherapy 2015, 7, 1159-1168. [PubMed: 26567563]

(52). Schneble EJ; Berry JS; Trappey FA; Clifton GT; Ponniah S; Mittendorf E; Peoples GE The HER2 peptide nelipepimut-S (E75) vaccine (NeuVax) in breast cancer patients at risk for recurrence: correlation of immunologic data with clinical response. Immunotherapy 2014, 6, 519-531. [PubMed: 24896623] 
a)

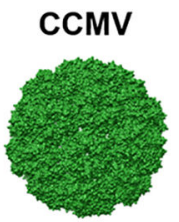

b)
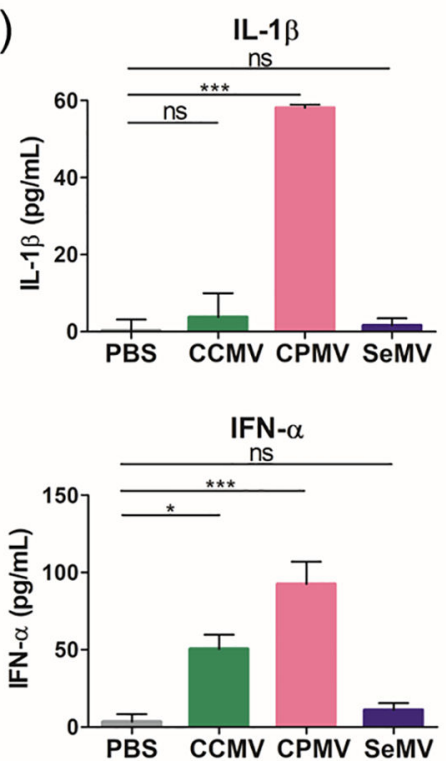
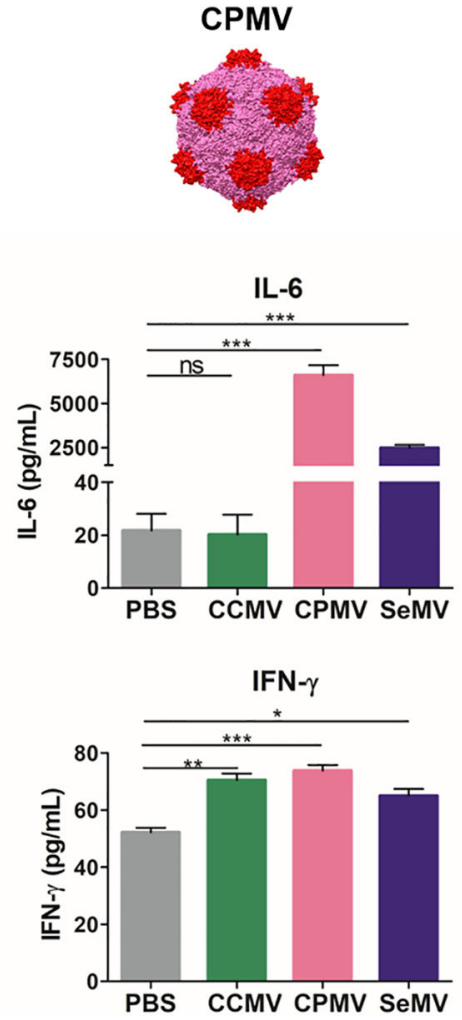

SeMV
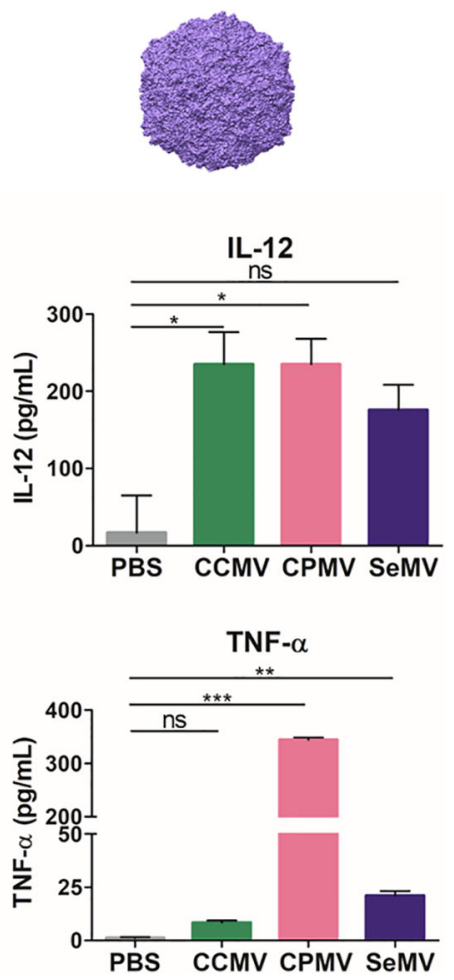

Figure 1.

(a) Plant virus nanoparticles (VNPs) CCMV, CPMV, and SeMV (images were created using Chimera software and PDB entries 1cwp for CCMV, 1ny7 for CPMV, and $1 \times 33$ for SeMV).

(b) CCMV, CPMV, and SeMV are inherently and differently immunogenic. BMDCs exposed to the VNPs for $18 \mathrm{~h}$ produced different levels of pro-inflammatory cytokines in vitro. Means of triplicates and standard deviations are shown. Statistical analysis by one-way analysis of variance (ANOVA) and Tukey's test: ns = not significant; ${ }^{*}, p<0.05 ; * *, p<$ $0.01 ; * * *, p<0.001$. 
a)

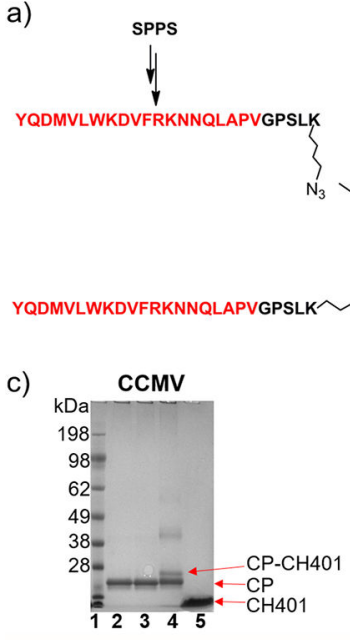

d)

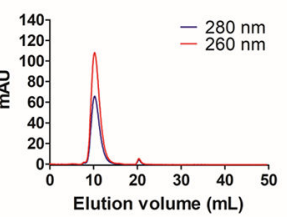

e)

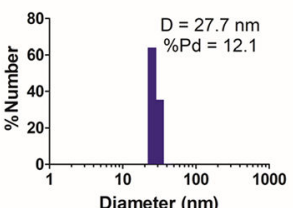

f)

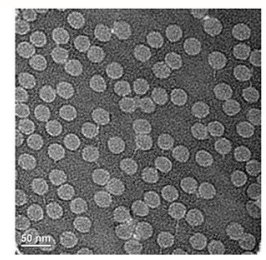

ㄴ. Lys

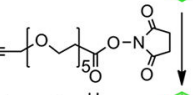

-

(i)

$\checkmark \mathrm{Cu}$<smiles>[AlH2]</smiles>
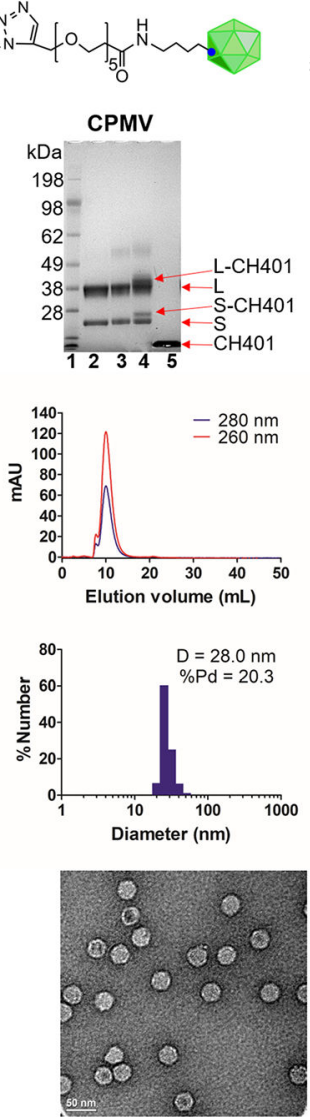

b)

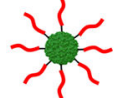

CCMV-CH401

CPMV-CH401

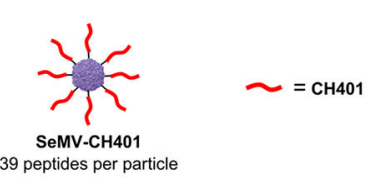

39 peptides per particle
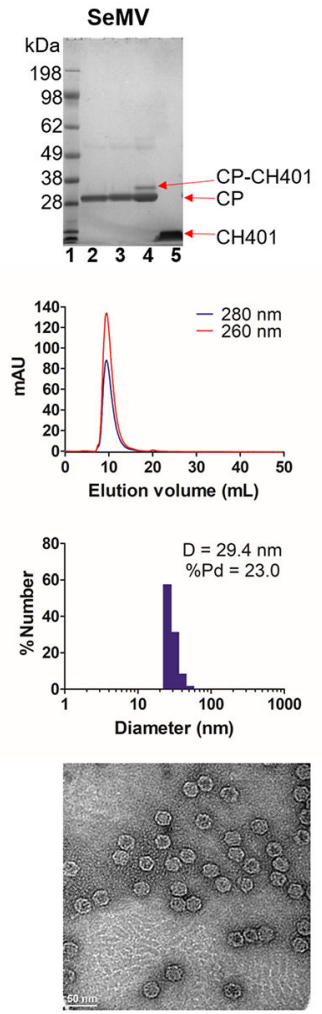

Figure 2.

Synthesis and characterization of VNP-CH401. (a) Conjugation of HER2 peptide CH401 to CCMV, CPMV, and SeMV using click chemistry. SPPS = solid-phase peptide synthesis. (b) VNP-based HER2 vaccine candidates. (c) SDS-PAGE: lane 1 (L1), ladder; L2, VNP; L3, VNP-alkyne; L4, VNP-CH401; L5, CH401. CP = capsid protein; $\mathrm{S}=$ small capsid protein of CPMV; L = large capsid protein of CPMV. (d) SEC analysis of VNP-CH401 using a Superose 6 size-exclusion column and Akta purifier. (e) Size distribution of VNP-CH401 measured by dynamic light scattering. $\mathrm{D}=$ diameter; $\mathrm{Pd}=$ polydispersity. (f) TEM images of negatively stained VNP-CH401 samples. 


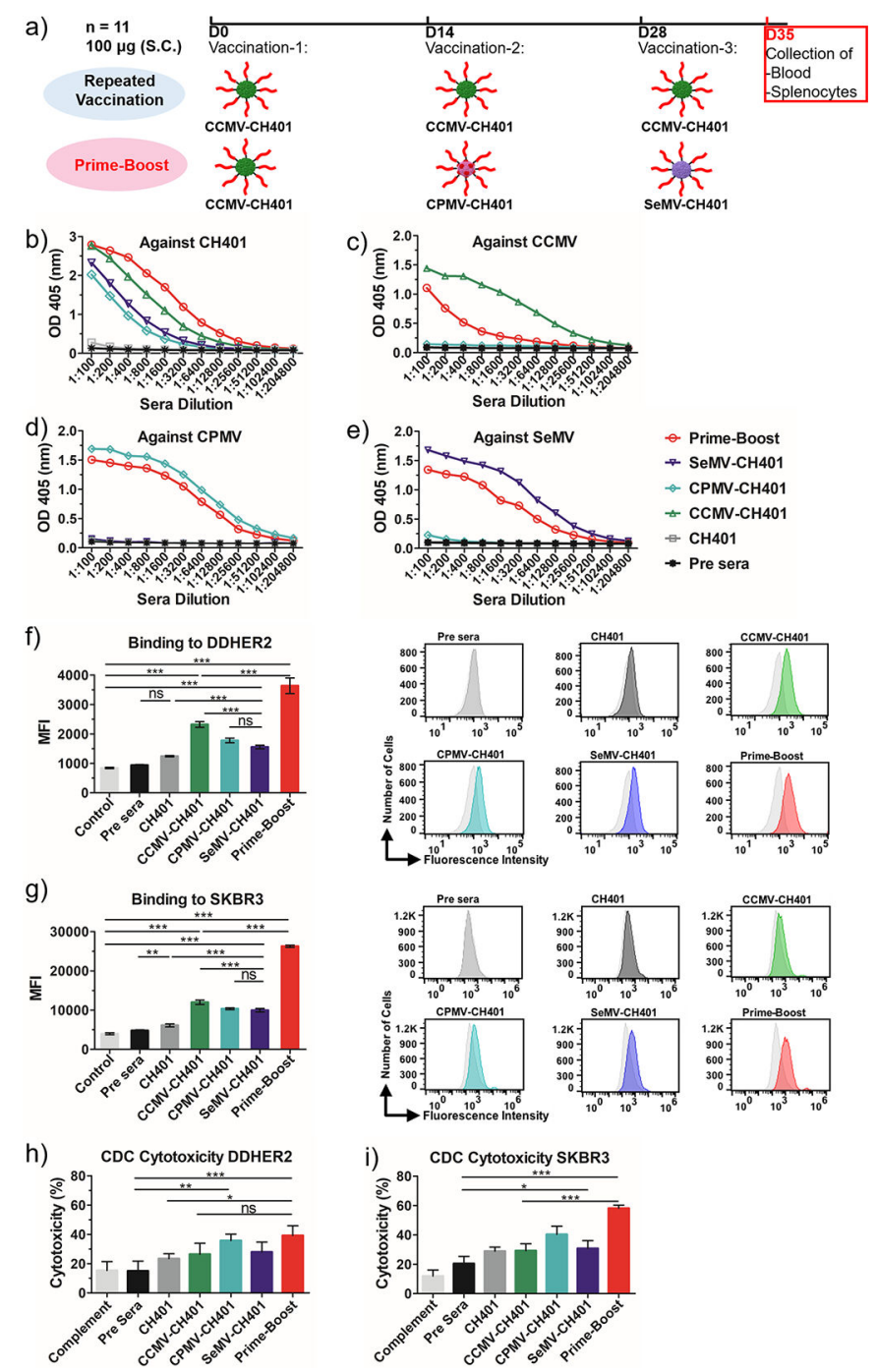

Figure 3.

(a) Schedule of homologous repeated boost and heterologous prime-boost strategies. (b-e) ELISA binding of the antisera IgG to (b) peptide, (c) CCMV, (d) CPMV, and (e) SeMV. (f, g) Flow cytometry analysis of the antisera (diluted 1:300) binding to (f) DDHER2 and (g) SKBR3 cancer cells. The mean fluorescence intensity (MFI) of triplicate experiments and standard deviations are shown. (h, i) MTT assay of complement-dependent cytotoxicity induced by the antisera in (h) DDHER2 and (i) SKBR3 cancer cells. Rabbit serum (diluted $1: 20)$ was used as the source of complement. Rabbit complement inactivated by heating at $65^{\circ} \mathrm{C}$ for $30 \mathrm{~min}$ was used as the control $(=100 \%$ cell viability). Cytotoxicity $(\%)=100-$ $($ experimental OD/control OD) $\times 100$. Mean cytotoxicities of quaduplicate experiments and standard deviations are shown. Statistical analysis by one-way ANOVA with Tukey's test: ns $=$ not significant; $*, p<0.05 ; * *, p<0.01 ; * * *, p<0.001$. 

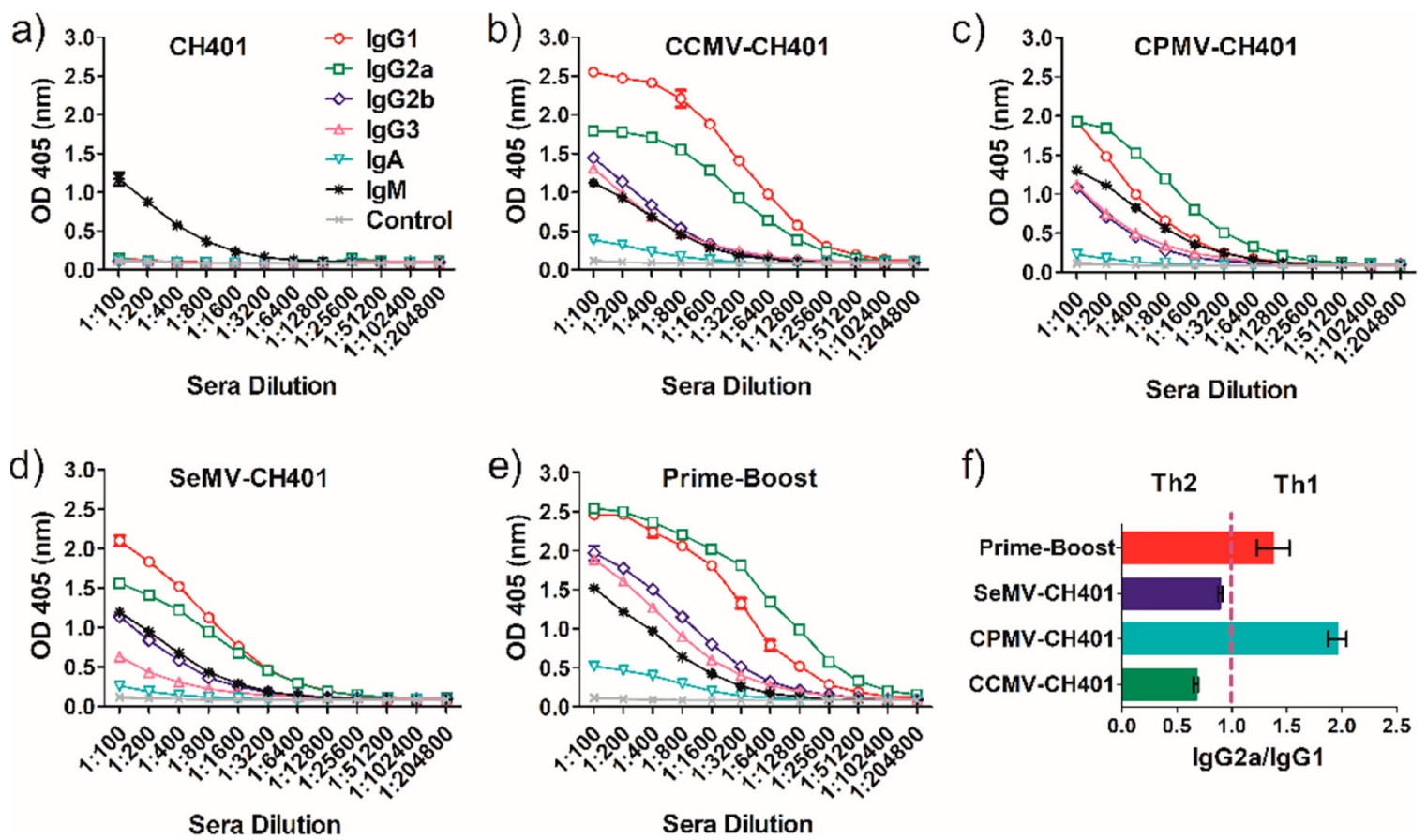

Sera Dilution

Sera Dilution

Figure 4.

(a-e) Antibody isotype analysis of the antisera induced by (a) CH401, (b) CCMV-CH401, (c) CPMV-CH401, (d) SeMV-CH401, and (e) prime-boost. (f) IgG2a/IgG1 ratio as determined by the OD value at a dilution of 1:1600. Mean ratios of duplicate experiments and standard deviations are shown. 

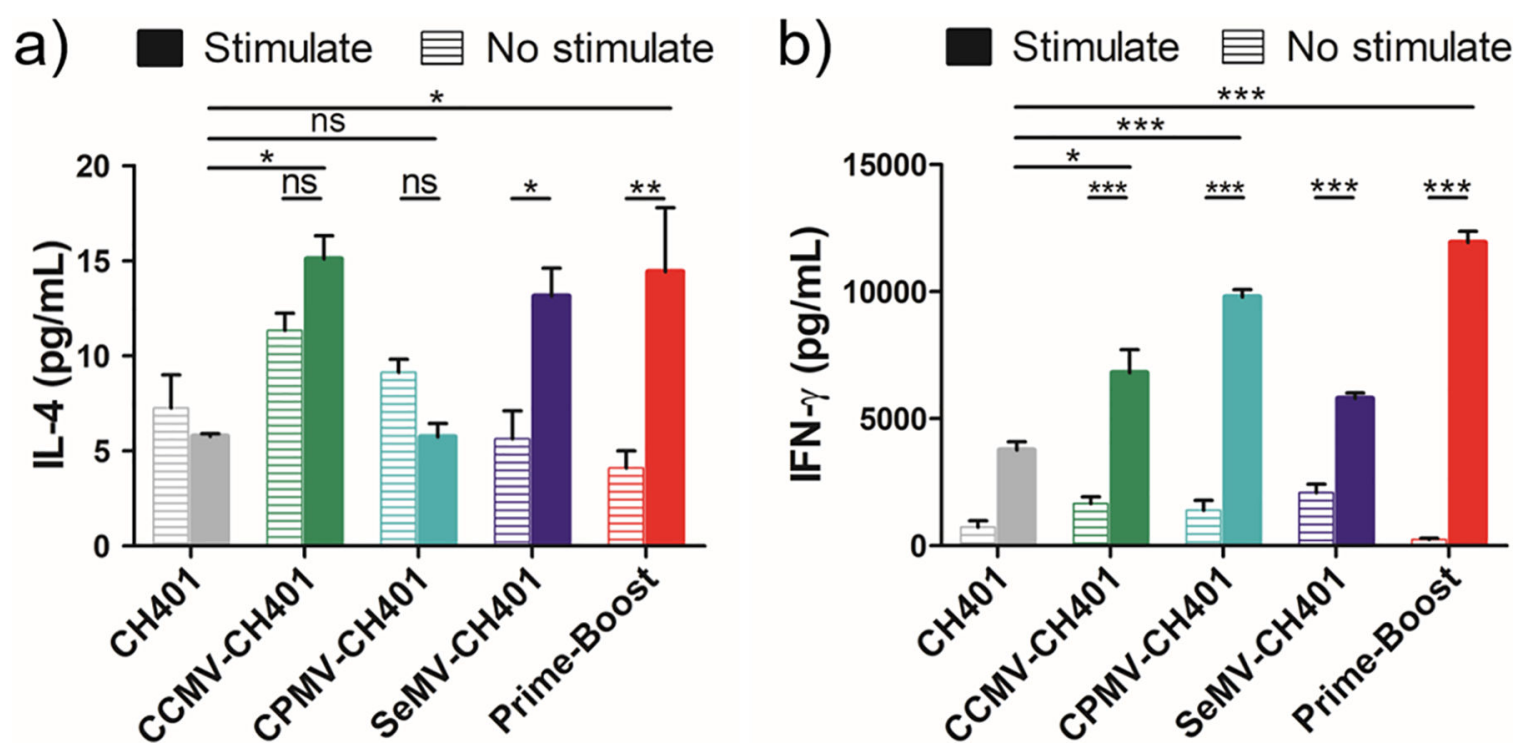

Figure 5.

(a) IL-4 and (b) IFN- $\gamma$ secretion from splenocytes isolated from immunized mice with and without exposure to $\mathrm{CH} 401$ peptide $(20 \mu \mathrm{g} / \mathrm{mL}, 18 \mathrm{~h})$ in vitro. Means of triplicates and standard deviations are shown. Statistical analysis between stimulated and unstimulated cells in each group by two-way ANOVA with Tukey's test: ns = not significant; ${ }^{*}, p<0.05$; ${ }^{*}, p$ $<0.01$; ***, $p<0.001$. 
a)

\section{Tumor size measure Every other day}

\begin{tabular}{|c|c|c|c|}
\hline $\begin{array}{l}\text { Day: }-42 \\
\text { 1st Vaccination } \\
n=8 \\
100 \mu \text { (S.C.) }\end{array}$ & $\begin{array}{l}\text { Day: } \mathbf{- 2 8} \\
\text { 2nd Vaccination }\end{array}$ & $\begin{array}{l}\text { Day: -14 } \\
\text { 3rd Vaccination }\end{array}$ & $\begin{array}{l}\text { Day: } 0 \\
\text { Tumor inoculation (S.C.) } \\
\text { Cancer cell: DDHER2 } \\
1 \times 10^{6} \text { cells/mouse }\end{array}$ \\
\hline
\end{tabular}

b)

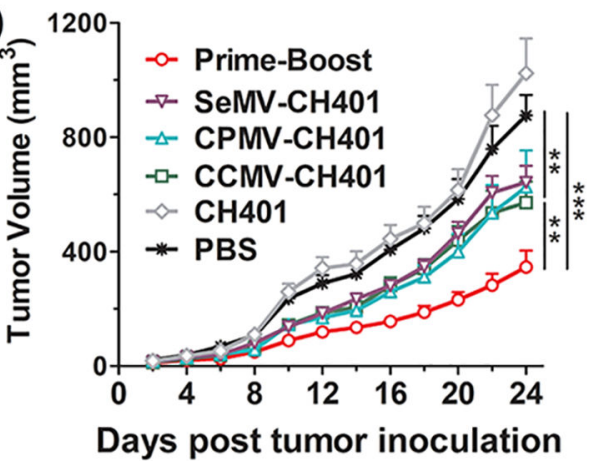

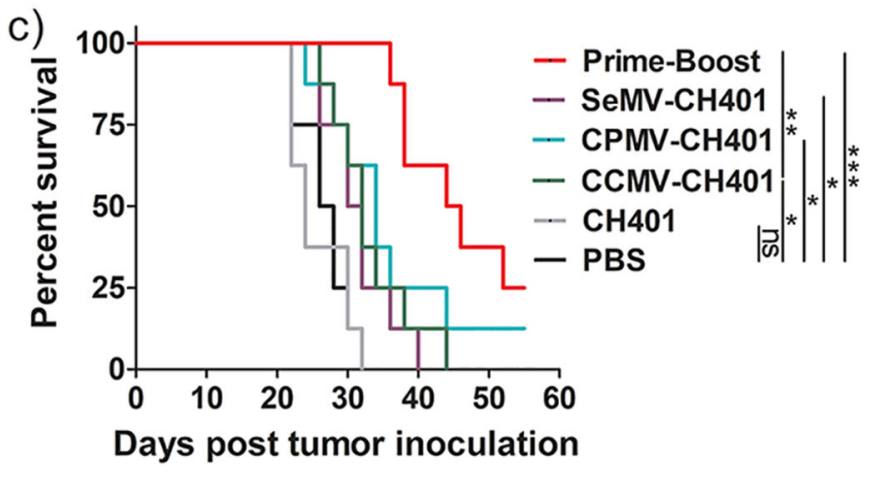

Figure 6.

(a) Schedule of vaccination and tumor inoculation. (b) Tumor growth curves. Mean tumor volumes and standard errors of the mean are shown. Statistical analysis by two-way ANOVA: **, $p<0.01$; ***, $p<0.001$. (c) Survival curves. Statistical analysis by log-rank (Mantel-Cox) test: $\mathrm{ns}=$ not significant; ${ }^{*}, p<0.05 ; * *, p<0.01 ; * * *, p<0.001$. 